\title{
Intuitionistic Fuzzy Subbialgebras and Duality
}

\author{
Wenjuan Chen ${ }^{1}$ and Bijan Davvaz ${ }^{2}$ \\ ${ }^{1}$ School of Mathematical Sciences, University of Jinan, Jinan, Shandong 250022, China \\ ${ }^{2}$ Department of Mathematics, Yazd University, Yazd 89195-741, Iran
}

Correspondence should be addressed to Wenjuan Chen; wjchenmath@gmail.com

Received 7 September 2013; Revised 22 January 2014; Accepted 6 February 2014; Published 18 March 2014

Academic Editor: Jianming Zhan

Copyright (C) 2014 W. Chen and B. Davvaz. This is an open access article distributed under the Creative Commons Attribution License, which permits unrestricted use, distribution, and reproduction in any medium, provided the original work is properly cited.

\begin{abstract}
We investigate connections between bialgebras and Atanassov's intuitionistic fuzzy sets. Firstly we define an intuitionistic fuzzy subbialgebra of a bialgebra with an intuitionistic fuzzy subalgebra structure and also with an intuitionistic fuzzy subcoalgebra structure. Secondly we investigate the related properties of intuitionistic fuzzy subbialgebras. Finally we prove that the dual of an intuitionistic fuzzy strong subbialgebra is an intuitionistic fuzzy strong subbialgebra.
\end{abstract}

\section{Introduction}

A bialgebra is, roughly speaking, an algebra on which there exists a dual structure called a coalgebra structure such that the two structures satisfy a compatibility relation. We know that group algebras, tensor algebras, symmetric algebras, enveloping algebras of Lie algebras, and so on are bialgebras [1]. Recently, the theory of bialgebras has many applications in several branches of both pure and applied sciences. So it is natural to ask whether we can extend the concept of bialgebras in uncertainty settings.

Uncertainty is an attribute of information and uncertain data are presented in various domains. The most appropriate theory for dealing with uncertainties is the theory of fuzzy sets developed by Zadeh [2]. Besides several generalizations of fuzzy sets, the intuitionistic fuzzy sets introduced by Atanassov [3,4] have been found to be highly useful to cope with imperfect and/or imprecise information. Atanassov's intuitionistic fuzzy sets are an intuitively straightforward extension of Zadeh's fuzzy sets: while a fuzzy set gives the degree of membership of an element in a given set, an Atanassov's intuitionistic fuzzy set gives both a degree of membership and a degree of nonmembership. Intuitionistic fuzzy set theory has been successfully applied in decision analysis and pattern recognition, in logic programming, and in medical diagnosis. And recently, many mathematicians have been involved in extending the concepts and results of abstract algebra to the broader framework of the intuitionistic fuzzy settings. For example, Biswas applied the concepts of intuitionistic fuzzy sets to the theory of groups and studied intuitionistic fuzzy subgroups of a group in [5]. Banerjee and Basnet studied intuitionistic fuzzy subrings and ideals of a ring in [6] and Hur et al. also discussed the concepts of the intuitionistic fuzzy ring in $[7,8]$. Further, Jun et al. investigated intuitionistic nil radicals of intuitionistic fuzzy ideals in rings in [9]; Akram and Dudek introduced intuitionistic fuzzy left k-ideals of semirings in [10]. Davvaz et al. [11] applied the notion of intuitionistic fuzzy sets to $H_{v^{-}}$ modules; Davvaz et al. [12-16] had studied the intuitionistic fuzzification of the concept of algebraic hyperstructures and investigated some of their properties; Chen and Zhang $[17,18]$ discussed Lie superalgebras in intuitionistic fuzzy settings and obtained some results. On the other hand, we had studied fuzzy subcoalgebras and fuzzy subbialgebras in [1921]. In this paper we will extend the concepts of bialgebras in intuitionistic fuzzy sets.

The structure of this paper is as follows. In Section 2, we recall some basic definitions and notations which will be used in what follows. In Section 3, we give the definition of an intuitionistic fuzzy subbialgebra and discuss the related properties. In Section 4, we define the duality of intuitionistic fuzzy subspaces which generalizes the related results in fuzzy settings. As the application, we prove that the duality of an 
intuitionistic fuzzy strong subbialgebra is an intuitionistic fuzzy strong subbialgebra. At last, a conclusion is presented.

\section{Preliminaries}

In this section, we recall some basic definitions and notations for the sake of completeness.

Definition 1. Let $V$ be a $k$-vector space. An intuitionistic fuzzy set (IFS for short) of $V$ is defined as an object having the form $A=\left\{\left\langle x, \mu_{A}(x), \nu_{A}(x)\right\rangle \mid x \in V\right\}$, where the functions $\mu_{A}: V \rightarrow[0,1]$ and $\nu_{A}: V \rightarrow[0,1]$ denote the degree of membership (namely, $\mu_{A}(x)$ ) and the degree of nonmembership (namely, $\nu_{A}(x)$ ) of each element $x \in V$ to the set $A$, respectively, and $0 \leq \mu_{A}(x)+v_{A}(x) \leq 1$ for each $x \in V$. For the sake of simplicity, we will use the symbol $A=\left(\mu_{A}, v_{A}\right)$ for the intuitionistic fuzzy set $A=\left\{\left\langle x, \mu_{A}(x), \nu_{A}(x)\right\rangle \mid x \in\right.$ $V\}$.

Definition 2. Let $A=\left(\mu_{A}, v_{A}\right)$ and $B=\left(\mu_{B}, v_{B}\right)$ be intuitionistic fuzzy sets of $k$-vector space $V$. Then one has the following:

(1) $A \subseteq B$ iff $\mu_{A}(x) \leq \mu_{B}(x)$ and $\nu_{A}(x) \geq \nu_{B}(x)$ for all $x \in V$,

(2) $A \cap B=\left\{\left\langle x, \mu_{A}(x) \wedge \mu_{B}(x), \nu_{A}(x) \vee v_{B}(x)\right\rangle \mid x \in V\right\}$,

(3) $A \cup B=\left\{\left\langle x, \mu_{A}(x) \vee \mu_{B}(x), \nu_{A}(x) \wedge \nu_{B}(x)\right\rangle \mid x \in V\right\}$,

(4) $\square A=\left\{\left\langle x, \mu_{A}(x), \mu_{A}^{c}(x)\right\rangle \mid x \in V\right\}$,

(5) $\diamond A=\left\{\left\langle x, v_{A}^{c}(x), v_{A}(x)\right\rangle \mid x \in V\right\}$.

Definition 3. Let $A=\left(\mu_{A}, v_{A}\right)$ and $B=\left(\mu_{B}, v_{B}\right)$ be intuitionistic fuzzy sets of $k$-vector space $V$. The intuitionistic sum of $A=\left(\mu_{A}, \nu_{A}\right)$ and $B=\left(\mu_{B}, \nu_{B}\right)$ is defined to the intuitionistic fuzzy set $A+B=\left(\mu_{A+B}, \nu_{A+B}\right)$ of $V$ given by

$$
\begin{aligned}
& \mu_{A+B}(x)= \begin{cases}\sup _{x=a+b}\left\{\mu_{A}(a) \wedge \mu_{B}(b)\right\} & \text { if } x=a+b \\
0 & \text { otherwise, }\end{cases} \\
& v_{A+B}(x)= \begin{cases}\inf _{x=a+b}\left\{\nu_{A}(a) \vee \nu_{B}(b)\right\} & \text { if } x=a+b \\
1 & \text { otherwise. }\end{cases}
\end{aligned}
$$

Definition 4. Let $A=\left(\mu_{A}, v_{A}\right)$ be an intuitionistic fuzzy set of $k$-vector space $V$. For any $x, y \in V$ and $\alpha, \beta \in k$, if it satisfies $\mu_{A}(\alpha x+\beta y) \geq \mu_{A}(x) \wedge \mu_{A}(y)$ and $\nu_{A}(\alpha x+\beta y) \leq \nu_{A}(x) \vee v_{A}(y)$, then $A=\left(\mu_{A}, v_{A}\right)$ is called an intuitionistic fuzzy subspace of $V$.

Definition 5. For any $t \in[0,1]$ and fuzzy subset $\mu$ of $V$, the set $U^{t}=\{x \in V \mid \mu(x) \geq t\}$ (resp., $L^{t}=\{x \in V \mid \mu(x) \leq t\}$ ) is called an upper (resp., lower) $t$-level cut of $\mu$ and $U^{\widehat{t}}=\{x \in$ $V \mid \mu(x)>t\}$ (resp., $L^{\widehat{t}}=\{x \in V \mid \mu(x)<t\}$ ) is called an upper (resp., lower) strong $t$-level cut of $\mu$.

Theorem 6. Let $A=\left(\mu_{A}, v_{A}\right)$ be an intuitionistic fuzzy set of $k$-vector space $V$ such that $\mu_{A}(V)$ with attained upper bound $\alpha>0$ and $\nu_{A}(V)$ with attained lower bound $\beta<1$ in $[0,1]$. Then the following statements are equivalent:
(1) $A=\left(\mu_{A}, v_{A}\right)$ is an intuitionistic fuzzy subspace of $V$,

(2) nonempty sets $U^{t}$ and $L^{t}$ are subspaces of $V$ for each $t \in[0, \alpha] \cap[\beta, 1]$,

(3) nonempty sets $U^{t}$ and $L^{t}$ are subspaces of $V$ for each $t \in \operatorname{Im} \mu_{A} \cap \operatorname{Im} v_{A}$,

(4) nonempty sets $U^{\hat{t}}$ and $L^{\hat{t}}$ are subspaces of $V$ for each $t \in[0, \alpha) \cap(\beta, 1]$.

Definition 7. Let $A=\left(\mu_{A}, v_{A}\right)$ be an intuitionistic fuzzy set of $k$-algebra $K$. For any $x, y \in K$ and $\alpha, \beta \in k$, if it satisfies the following conditions:

(1) $\mu_{A}(\alpha x+\beta y) \geq \mu_{A}(x) \wedge \mu_{A}(y)$ and $v_{A}(\alpha x+\beta y) \leq$ $v_{A}(x) \vee v_{A}(y)$

(2) $\mu_{A}(x y) \geq \mu_{A}(x) \wedge \mu_{A}(y)$ and $\nu_{A}(x y) \leq v_{A}(x) \vee v_{A}(y)$,

then $A=\left(\mu_{A}, v_{A}\right)$ is called an intuitionistic fuzzy subalgebra of $K$.

Definition 8. Let $A=\left(\mu_{A}, v_{A}\right)$ be an intuitionistic fuzzy set of $k$-algebra $K$. For any $x, y \in K$ and $\alpha, \beta \in k$, if it satisfies the following conditions:

(1) $\mu_{A}(\alpha x+\beta y) \geq \mu_{A}(x) \wedge \mu_{A}(y)$ and $\nu_{A}(\alpha x+\beta y) \leq$ $v_{A}(x) \vee v_{A}(y)$,

(2) $\mu_{A}(x y) \geq \mu_{A}(x) \vee \mu_{A}(y)$ and $\nu_{A}(x y) \leq v_{A}(x) \wedge v_{A}(y)$,

then $A=\left(\mu_{A}, v_{A}\right)$ is called an intuitionistic fuzzy ideal of $K$.

Definition 9 (see [21]). Let $A=\left(\mu_{A}, v_{A}\right)$ be an intuitionistic fuzzy set of $k$-coalgebra $C$. For any $x, y \in C, \Delta(x)=$ $\sum_{i=1, n} x_{i 1} \otimes x_{i 2}$ and $\alpha, \beta \in k$, if it satisfies the following conditions:

(1) $\mu_{A}(\alpha x+\beta y) \geq \mu_{A}(x) \wedge \mu_{A}(y)$ and $\nu_{A}(\alpha x+\beta y) \leq$ $v_{A}(x) \vee v_{A}(y)$

(2) $\mu_{A}(x) \leq \mu_{A}\left(x_{i 1}\right) \wedge \mu_{A}\left(x_{i 2}\right)$ and $v_{A}(x) \geq v_{A}\left(x_{i 1}\right) \vee$ $\nu_{A}\left(x_{i 2}\right)$, for all $i$,

then $A=\left(\mu_{A}, v_{A}\right)$ is called intuitionistic fuzzy subcoalgebra of C.

Definition 10 (see [1]). A bialgebra is a $k$-vector space $H$, endowed with an algebra structure $(H, M, u)$ and with a coalgebra structure $(H, \Delta, \varepsilon)$ such that $M$ and $u$ are morphisms of coalgebras (equivalently, $\Delta$ and $\varepsilon$ are morphisms of algebras). In this paper, we always denote $H$ as a bialgebra over field $k$.

Definition 11. Let $H$ be a bialgebra. A $k$-subspace $K$ of $H$ is called a subbialgebra if it is endowed with a subalgebra structure and with a subcoalgebra structure.

Remark 12. Let $H$ be a bialgebra. Then $(H, \Delta, \varepsilon)$ is a coalgebra. For an element $x \in H, \Delta(x)=\sum_{i=1, n} x_{i 1} \otimes x_{i 2}$. In this paper, we require that $\left\{x_{i 1}\right\},\left\{x_{i 2}\right\}$ are linearly independent, respectively. In this case, the decomposition of $\Delta(x)$ is unique. 


\section{Intuitionistic Fuzzy Subbialgebras of Bialgebras}

At first, we give the following.

Definition 13. Let $A=\left(\mu_{A}, \nu_{A}\right)$ be an intuitionistic fuzzy set of bialgebra $H$. Then $A=\left(\mu_{A}, \nu_{A}\right)$ is called an intuitionistic fuzzy subbialgebra of $H$, if, for any $x, y \in H$ and $\alpha, \beta \in k$, the following conditions are satisfied:

(1) $A=\left(\mu_{A}, v_{A}\right)$ is an intuitionistic fuzzy subspace of $H$,

(2) $\mu_{A}(x y) \geq \mu_{A}(x) \wedge \mu_{A}(y)$ and $\nu_{A}(x y) \leq \nu_{A}(x) \vee v_{A}(y)$,

(3) $\mu(x) \leq \mu\left(x_{i 1}\right) \wedge \mu\left(x_{i 2}\right)$ and $\nu(x) \geq \nu\left(x_{i 1}\right) \vee \nu\left(x_{i 2}\right)$, for all $i$.

Remark 14. If $A=\left(\mu_{A}, v_{A}\right)$ satisfies (1) and (2), then $A=$ $\left(\mu_{A}, v_{A}\right)$ is an intuitionistic fuzzy subalgebra of $H$; if $A=$ $\left(\mu_{A}, v_{A}\right)$ satisfies (1) and (3), then $A=\left(\mu_{A}, v_{A}\right)$ is an intuitionistic fuzzy subcoalgebra of $H$. So an intuitionistic fuzzy subbialgebra is with an intuitionistic fuzzy subalgebra structure and also with an intuitionistic fuzzy subcoalgebra structure.

Definition 15. Let $A=\left(\mu_{A}, v_{A}\right)$ be an intuitionistic fuzzy set of bialgebra $H$. Then $A=\left(\mu_{A}, \nu_{A}\right)$ is called an intuitionistic fuzzy strong subbialgebra of $H$, if, for any $x, y \in H$ and $\alpha$, $\beta \in k$, the following conditions are satisfied:

(1) $A=\left(\mu_{A}, v_{A}\right)$ is an intuitionistic fuzzy subspace of $H$,

(2) $\mu_{A}(x y) \geq \mu_{A}(x) \vee \mu_{A}(y)$ and $\nu_{A}(x y) \leq \nu_{A}(x) \wedge \nu_{A}(y)$,

(3) $\mu(x) \leq \mu\left(x_{i 1}\right) \wedge \mu\left(x_{i 2}\right)$ and $\nu(x) \geq \nu\left(x_{i 1}\right) \vee v\left(x_{i 2}\right)$, for all $i$.

Remark 16. If $A=\left(\mu_{A}, v_{A}\right)$ satisfies (1) and (2), then $A=$ $\left(\mu_{A}, \nu_{A}\right)$ is an intuitionistic fuzzy ideal of $H$. So an intuitionistic fuzzy strong subbialgebra is with an intuitionistic fuzzy ideal structure and with an intuitionistic fuzzy subcoalgebra structure. In fact, it is an intuitionistic fuzzy subbialgebra.

Example 17. The field $k$ with its algebra structure and with the canonical coalgebra structure $(\Delta(x)=x \otimes 1$ and $\varepsilon=I)$ is a bialgebra (see [1]).

We define $A=\left(\mu_{A}, \nu_{A}\right): k \rightarrow[0,1]$ by

$$
\begin{aligned}
& \mu_{A}(x)= \begin{cases}0.3 & \text { if } x \in k \backslash\{0,1\} \\
0.5 & x=1 \\
1 & x=0,\end{cases} \\
& \nu_{A}(x)= \begin{cases}0.4 & \text { if } x \in k \backslash\{0,1\} \\
0.2 & x=1 \\
0 & x=0 .\end{cases}
\end{aligned}
$$

Then $A=\left(\mu_{A}, \nu_{A}\right)$ is an intuitionistic fuzzy subbialgebra of $k$. However, $A=\left(\mu_{A}, v_{A}\right)$ is not an intuitionistic fuzzy strong subbialgebra, since $\mu_{A}(x \cdot 1)=\mu_{A}(x)=0.3<\mu_{A}(x) \vee$ $\mu_{A}(1)=0.5$ for $x \in k \backslash\{0,1\}$.

Example 18. If $G$ is a monoid, then the semigroup algebra $k G$ endowed with a coalgebra structure as $\Delta(g)=g \otimes g$ and $\varepsilon(g)=1$ for any $g \in G$ is a bialgebra (see [1]).
We define $A=\left(\mu_{A}, v_{A}\right): k G \rightarrow[0,1]$ by $\mu_{A}(x)=$ $\bigwedge_{i \in N} \mu_{A}\left(s_{i}\right)$ and $\nu_{A}(x)=\bigvee_{i \in N} \nu_{A}\left(s_{i}\right)$ for $0 \neq x=\sum_{i \in N} k_{i} s_{i}$. If $x=0$, we define $\mu_{A}(0)=1$ and $\nu_{A}(0)=0$. Then $A=\left(\mu_{A}, \nu_{A}\right)$ is an intuitionistic fuzzy subbialgebra of $k G$.

Lemma 19. If $A=\left(\mu_{A}, v_{A}\right)$ is an intuitionistic fuzzy subbialgebra of $H$, then so is $\square A=\left(\mu_{A}, \mu_{A}^{c}\right)$.

Lemma 20. If $A=\left(\mu_{A}, v_{A}\right)$ is an intuitionistic fuzzy subbialgebra of $H$, then so is $\nabla A=\left(\nu_{A}^{c}, v_{A}\right)$.

Combining Lemmas 19 and 20, it is easy to prove the following theorem.

Theorem 21. $A=\left(\mu_{A}, v_{A}\right)$ is an intuitionistic fuzzy subbialgebra of $H$ if and only if $\square A$ and $\diamond A$ are intuitionistic fuzzy subbialgebras.

The following theorems explain the relationship between intuitionistic fuzzy subbialgebras and subbialgebras. The proof can be imitated by $[19,22]$. Here, we omit.

Theorem 22. If $A=\left(\mu_{A}, v_{A}\right)$ is an intuitionistic fuzzy subbialgebra of $H$, then the sets $U^{t}$ and $L^{t}$ are subbialgebras of $H$ for every $t \in \operatorname{Im} \mu_{A} \cap \operatorname{Im} v_{A}$.

Theorem 23. If $A=\left(\mu_{A}, v_{A}\right)$ is an intuitionistic fuzzy set of $H$ such that all nonempty level sets $U^{t}$ and $L^{t}$ are subbialgebras of $H$, then $A=\left(\mu_{A}, \nu_{A}\right)$ is an intuitionistic fuzzy subbialgebra of $H$.

Theorem 24. Let $A=\left(\mu_{A}, v_{A}\right)$ and $B=\left(\mu_{B}, v_{B}\right)$ be intuitionistic fuzzy subbialgebras of bialgebra $H$ such that $\mu_{A}(0)=\mu_{B}(0)$ and $\nu_{A}(0)=\nu_{B}(0)$. Then $A+B=\left(\mu_{A+B}, \nu_{A+B}\right)$ is also an intuitionistic fuzzy subbialgebra of $H$.

Proof. Obviously $A+B=\left(\mu_{A+B}, v_{A+B}\right)$ is an intuitionistic fuzzy subspace of $H$. We only show that $\mu_{A+B}(x y) \geq \mu_{A+B}(x) \wedge$ $\mu_{A+B}(y), v_{A+B}(x y) \leq v_{A+B}(x) \vee v_{A+B}(y)$ and $\mu_{A+B}(x) \leq$ $\mu_{A+B}\left(x_{i 1}\right) \wedge \mu_{A+B}\left(x_{i 2}\right), v_{A+B}(x) \geq v_{A+B}\left(x_{i 1}\right) \vee v_{A+B}\left(x_{i 2}\right)$ for $x, y \in H$.

Let $x=a+b$ and $y=c+d \in H$. Then $x y=a c+a d+$ $b c+b d$. Otherwise, $x \neq a+b$ or $y \neq c+d$; the result can be proved similarly. We have

$$
\begin{aligned}
\mu_{A+B}(x y) & =\sup _{x y=m+n}\left\{\mu_{A}(m) \wedge \mu_{B}(n)\right\} \\
\geq & \sup _{x y=a c+a d+b c+b d}\left\{\mu_{A}(a c+a d) \wedge \mu_{B}(b c+b d)\right\} \\
\geq & \sup _{x y=a c+a d+b c+b d}\left\{\mu_{A}(a c) \wedge \mu_{A}(a d)\right. \\
\geq & \left.\wedge \mu_{B}(b c) \wedge \mu_{B}(b d)\right\} \\
& \left\{\sup _{x y=a c+a d+b c+b d}(a) \wedge \mu_{A}(c) \wedge \mu_{A}(d)\right. \\
& \left.\wedge \mu_{B}(b) \wedge \mu_{B}(c) \wedge \mu_{B}(d)\right\}
\end{aligned}
$$




$$
\begin{aligned}
& =\sup _{x y=a c+a d+b c+b d}\left\{\left(\mu_{A}(a) \wedge \mu_{B}(b)\right)\right. \\
& \wedge\left(\mu_{A}(c) \wedge \mu_{B}(d)\right) \\
& \left.\wedge\left(\mu_{A}(d) \wedge \mu_{B}(c)\right)\right\} \\
& =\mu_{A+B}(x) \wedge \mu_{A+B}(y) \wedge \mu_{A+B}(y) \\
& =\mu_{A+B}(x) \wedge \mu_{A+B}(y), \\
& v_{A+B}(x y)=\inf _{x y=m+n}\left\{v_{A}(m) \vee v_{B}(n)\right\} \\
& \leq \inf _{x y=a c+a d+b c+b d}\left\{v_{A}(a c+a d) \vee v_{B}(b c+b d)\right\} \\
& \leq \inf _{x y=a c+a d+b c+b d}\left\{v_{A}(a c) \vee v_{A}(a d) \vee v_{B}(b c)\right. \\
& \left.\vee v_{B}(b d)\right\} \\
& \leq \inf _{x y=a c+a d+b c+b d}\left\{v_{A}(a) \vee v_{A}(c) \vee v_{A}(d)\right. \\
& \left.\vee v_{B}(b) \vee v_{B}(c) \vee v_{B}(d)\right\} \\
& =\inf _{x y=a c+a d+b c+b d}\left\{\left(v_{A}(a) \vee v_{B}(b)\right)\right. \\
& \vee\left(\nu_{A}(c) \vee v_{B}(d)\right) \\
& \left.\vee\left(\nu_{A}(d) \vee v_{B}(c)\right)\right\} \\
& =v_{A+B}(x) \vee v_{A+B}(y) \vee v_{A+B}(y) \\
& =v_{A+B}(x) \vee v_{A+B}(y) \text {. }
\end{aligned}
$$

On the other hand, let $x=a+b \in H$. Then

$$
\begin{aligned}
\sum_{i=1, n} x_{i 1} \otimes x_{i 2} & =\Delta(x)=\Delta(a+b)=\Delta(a)+\Delta(b) \\
& =\sum_{s=1, n} a_{s 1} \otimes a_{s 2}+\sum_{t=1, n} b_{t 1} \otimes b_{t 2} .
\end{aligned}
$$

Hence we have

$$
\begin{aligned}
\mu_{A+B}(x)= & \sup _{x=a+b}\left\{\mu_{A}(a) \wedge \mu_{B}(b)\right\} \\
\leq & \sup _{x=a+b}\left\{\left(\mu_{A}\left(a_{s 1}\right) \wedge \mu_{A}\left(a_{s 2}\right)\right)\right. \\
\qquad & \left.\wedge\left(\mu_{B}\left(b_{t 1}\right) \wedge \mu_{B}\left(b_{t 2}\right)\right)\right\} \\
= & \sup _{x=a+b}\left\{\mu_{A}\left(a_{s 1}\right) \wedge \mu_{B}(0) \wedge \mu_{A}(0) \wedge \mu_{B}\left(b_{t 1}\right)\right\} \\
& \wedge \sup _{x=a+b}\left\{\mu_{A}\left(a_{s 2}\right) \wedge \mu_{B}(0) \wedge \mu_{A}(0) \wedge \mu_{B}\left(b_{t 2}\right)\right\} \\
\leq & \left(\mu_{A+B}\left(a_{s 1}\right) \wedge \mu_{A+B}\left(b_{t 1}\right)\right) \\
& \wedge\left(\mu_{A+B}\left(a_{s 2}\right) \wedge \mu_{A+B}\left(b_{t 2}\right)\right) \\
\leq & \mu_{A+B}\left(x_{i 1}\right) \wedge \mu_{A+B}\left(x_{i 2}\right)
\end{aligned}
$$

$$
\begin{aligned}
v_{A+B}(x)= & \inf _{x=a+b}\left\{v_{A}(a) \vee v_{B}(b)\right\} \\
\geq & \inf _{x=a+b}\left\{\left(v_{A}\left(a_{s 1}\right) \vee v_{A}\left(a_{s 2}\right)\right) \vee\left(v_{B}\left(b_{t 1}\right) \vee v_{B}\left(b_{t 2}\right)\right)\right\} \\
= & \inf _{x=a+b}\left\{v_{A}\left(a_{s 1}\right) \vee v_{B}(0) \vee v_{A}(0) \vee v_{B}\left(b_{t 1}\right)\right\} \\
& \vee \inf _{x=a+b}\left\{v_{A}\left(a_{s 2}\right) \vee v_{B}(0) \vee v_{A}(0) \vee v_{B}\left(b_{t 2}\right)\right\} \\
\geq & \left(v_{A+B}\left(a_{s 1}\right) \vee v_{A+B}\left(b_{t 1}\right)\right) \\
& \vee\left(v_{A+B}\left(a_{s 2}\right) \vee v_{A+B}\left(b_{t 2}\right)\right) \\
\geq & v_{A+B}\left(x_{i 1}\right) \vee v_{A+B}\left(x_{i 2}\right) .
\end{aligned}
$$

Therefore, $A+B=\left(\mu_{A+B}, v_{A+B}\right)$ is an intuitionistic fuzzy subbialgebra of $H$.

Theorem 25. Let $A=\left(\mu_{A}, v_{A}\right)$ and $B=\left(\mu_{B}, \nu_{B}\right)$ be any intuitionistic fuzzy subbialgebras of bialgebra $H$. Then $A \cap B=$ $\left(\mu_{A \cap B}, v_{A \cap B}\right)$ is also an intuitionistic fuzzy subbialgebra of $H$.

Corollary 26. Let $\left(A_{i}\right)_{i \in N}$ be a family of intuitionistic fuzzy subbialgebras of bialgebra $H$. Then $\bigcap_{i \in N} A_{i}=$ $\left(\mu_{\bigcap_{i \in N} A_{i}}, \nu_{\bigcap_{i \in N} A_{i}}\right)$ is also an intuitionistic fuzzy subbialgebra of $H$.

Let $H$ and $L$ be two bialgebras. The $k$-linear map $f$ : $H \rightarrow L$ is called a morphism of bialgebras if it is a morphism of algebras and a morphism of coalgebras between the underlying algebras, respectively, coalgebras of the two bialgebras.

Definition 27. Let $f$ be a map from bialgebra $H$ to bialgebra $L$. If $A=\left(\mu_{A}, v_{A}\right)$ and $B=\left(\mu_{B}, \nu_{B}\right)$ are intuitionistic fuzzy sets of $H$ and $L$, respectively, then the preimage of $B=\left(\mu_{B}, \nu_{B}\right)$ under $f$ is defined to be an intuitionistic fuzzy set $f^{-1}(B)=$ $\left(\mu_{f^{-1}(B)}, v_{f^{-1}(B)}\right)$ where $\mu_{f^{-1}(B)}(x)=\mu_{B}(f(x))$ and $v_{f^{-1}(B)}(x)=$ $\nu_{B}(f(x))$ for any $x \in H$ and the image of $A=\left(\mu_{A}, v_{A}\right)$ under $f$ is defined to be an intuitionistic fuzzy set $f(A)=$ $\left(\mu_{f(A)}, v_{f(A)}\right)$ where

$$
\begin{aligned}
& \mu_{f(A)}(y)= \begin{cases}\sup _{x \in f^{-1}(y)}\left\{\mu_{A}(x)\right\} & \text { if } y \in f(H) \\
0 & \text { if } y \notin f(H),\end{cases} \\
& v_{f(A)}(y)= \begin{cases}\inf _{x \in f^{-1}(y)}\left\{\nu_{A}(x)\right\} & \text { if } y \in f(H) \\
1 & \text { if } y \notin f(H) .\end{cases}
\end{aligned}
$$

Proposition 28. Let $f: H \rightarrow L$ be a homomorphism of bialgebras. If $A=\left(\mu_{A}, v_{A}\right)$ is an intuitionistic fuzzy subbialgebra of $L$, then $f^{-1}(A)=\left(\mu_{f^{-1}(A)}, \nu_{f^{-1}(A)}\right)$ is an intuitionistic fuzzy subbialgebra of $H$.

Proposition 29. Let $f: H \rightarrow L$ be a homomorphism of bialgebras. If $A=\left(\mu_{A}, v_{A}\right)$ is an intuitionistic fuzzy subbialgebra of $H$, then $f(A)=\left(\mu_{f(A)}, v_{f(A)}\right)$ is an intuitionistic fuzzy subbialgebra of $L$. 
Proposition 30. Let $A=\left(\mu_{A}, v_{A}\right)$ and $B=\left(\mu_{B}, \nu_{B}\right)$ be any intuitionistic fuzzy subbialgebras of bialgebra $H$ and let $f: H \rightarrow L$ be a homomorphism. If $\mu_{A}(0)=\mu_{B}(0)$ and $v_{A}(0)=v_{B}(0)$, then $f(A+B)$ is also an intuitionistic fuzzy subbialgebra of $L$ and $f(A+B)=f(A)+f(B)$.

\section{The Duality of Intuitionistic Fuzzy Sub-Bialgebras}

The following is well known.

Proposition 31 (see [1]). Let $H$ be a finite dimensional bialgebra. Then $H^{*}$, together with the algebra structure which is dual to the coalgebra structure of $H$ and with the coalgebra structure which is dual to the algebra structure of $H$, is a bialgebra, which is called the dual bialgebra of $H$.

Let $A=\left(\mu_{A}, \nu_{A}\right)$ be an intuitionistic fuzzy subspace of $k$ vector space $V$. Then $0 \leq \mu_{A}(x)+\nu_{A}(x) \leq 1$ for any $x \in V$, which implies $0 \leq \sup _{x \in V}\left\{\mu_{A}(x)\right\}+\inf _{x \in V}\left\{v_{A}(x)\right\} \leq 1$. We give the definition of the dual of intuitionistic fuzzy subspaces in the following [23].

Definition 32 (see [23]). Let $A=\left(\mu_{A}, v_{A}\right)$ be an intuitionistic fuzzy subspace of $k$-vector space $V$.

Define $A^{*}=\left(\mu_{A^{*}}, v_{A^{*}}\right)$ where

$\mu_{A^{*}}: V^{*} \longrightarrow[0,1]$ by

$\mu_{A^{*}}(f)= \begin{cases}\inf \left\{v_{A}(x) \mid x \in V, f(x) \neq 0\right\} & \text { if } f \neq 0 \\ \sup \left\{v_{A}(x) \mid x \in V\right\} & \text { if } f=0,\end{cases}$

$v_{A^{*}}: V^{*} \longrightarrow[0,1]$ by

$v_{A^{*}}(f)= \begin{cases}\sup \left\{\mu_{A}(x) \mid x \in V, f(x) \neq 0\right\} & \text { if } f \neq 0 \\ \inf \left\{\mu_{A}(x) \mid x \in V\right\} & \text { if } f=0 .\end{cases}$ $V^{*}$.

Obviously, $A^{*}=\left(\mu_{A^{*}}, v_{A^{*}}\right)$ is an intuitionistic fuzzy set of

Remark 33. (1) If $A=\square A$, then $A^{*}=\diamond A^{*}$.

(2) If $A=\diamond A$, then $A^{*}=\square A^{*}$.

Theorem 34. The intuitionistic fuzzy set $A^{*}=\left(\mu_{A^{*}}, v_{A^{*}}\right)$ is an intuitionistic fuzzy subspace of $V^{*}$.

Proof. Since $\mu_{\mathrm{A}^{*}}(0)$ is the upper bound of $\mu_{A^{*}}\left(V^{*}\right)$ and $\nu_{A^{*}}(0)$ is the lower bound of $v_{A^{*}}\left(V^{*}\right)$, by Theorem 6 , it suffices to show that the nonempty sets $U^{t}=\left\{f \in V^{*} \mid \mu_{A^{*}}(f) \geq t\right\}$ and $L^{t}=\left\{f \in V^{*} \mid v_{A^{*}}(f) \leq t\right\}$ are subspaces of $V^{*}$ for all $t \in\left[v_{A^{*}}(0), \mu_{A^{*}}(0)\right]$.

Case 1. $\mu_{A}(0) \leq \mu_{A^{*}}(0)$ and $v_{A}(0) \geq v_{A^{*}}(0)$.

First, we show $U^{t}=\left(H^{\hat{t}}\right)^{\perp}$ in which $H^{\hat{t}}=\{x \in V \mid$ $\left.v_{A}(x)<t\right\}$ and $L^{t}=\left(K^{\widehat{t}}\right)^{\perp}$ in which $K^{\widehat{t}}=\left\{x \in V \mid \mu_{A}(x)>t\right\}$, for all $t \in\left(\nu_{A}(0), \mu_{A}(0)\right)$. $\{0\}$.

Indeed, $U^{t}=\left\{f \in V^{*} \mid \inf \left\{v_{A}(x) \mid x \in V, f(x) \neq 0\right\} \geq t\right\} \cup$

$$
\text { If } g \in\left(H^{\widehat{t}}\right)^{\perp} \text {, then } g\left(H^{\widehat{t}}\right)=0 \text { and } g \in U^{t} \text {. }
$$

If $g \in V^{*} \backslash\left(H^{\widehat{t}}\right)^{\perp}$, then $g\left(H^{\widehat{t}}\right) \neq 0$. So there exists $x \in$ $V$, such that $g(x) \neq 0$ and $\nu_{A}(x)<t$. Then $g \notin U^{t}$.

Also, $L^{t}=\left\{f \in V^{*} \mid \sup \left\{\mu_{A}(x) \mid x \in V, f(x) \neq 0\right\} \leq\right.$ $t\} \cup\{0\}$.

If $g \in\left(K^{\widehat{t}}\right)^{\perp}$, then $g\left(K^{\widehat{t}}\right)=0$ and $g \in L^{t}$.

If $g \in V^{*} \backslash\left(K^{\widehat{t}}\right)^{\perp}$, then $g\left(K^{\widehat{t}}\right) \neq 0$. So there exists $x \in V$ such that $\mu_{A}(x)>t$ and $g(x) \neq 0$. Then $g \notin L^{t}$.

Second, if $\nu_{A^{*}}(0) \leq t \leq v_{A}(0)$ and $t \in \operatorname{Im} \mu_{A^{*}}$, then $\inf \left\{v_{A}(x) \mid x \in V, f(x) \neq 0\right\} \geq \inf \left\{v_{A}(V)\right\}=v_{A}(0) \geq t$, so $U^{t}=\left\{f \in V^{*} \mid \inf \left\{v_{A}(x) \mid x \in V, f(x) \neq 0\right\} \geq t\right\} \cup\{0\}=V^{*}$ and $L^{t}=\{0\}$.

If $\mu_{A}(0) \leq t \leq \mu_{A^{*}}(0)$ and $t \in \operatorname{Im} v_{A^{*}}$, then $\sup \left\{\mu_{A}(x) \mid\right.$ $x \in V, f(x) \neq 0\} \leq \sup \left\{\mu_{A}(V)\right\}=\mu_{A}(0) \leq t$, so $L^{t}=\left\{f \in V^{*} \mid\right.$ $\left.\sup \left\{\mu_{A}(x) \mid x \in V, f(x) \neq 0\right\} \leq t\right\} \cup\{0\}=V^{*}$ and $U^{t}=\{0\}$.

Cases 2-4 can be proved similarly as in Case 1 .

Case 2. $\mu_{A}(0) \geq \mu_{A^{*}}(0)$ and $\nu_{A}(0) \leq v_{A^{*}}(0)$.

We show $U^{t}=\left(H^{\widehat{t}}\right)^{\perp}$ and $L^{t}=\left(K^{\widehat{t}}\right)^{\perp}$, for all $t \in$ $\left(v_{A^{*}}(0), \mu_{A^{*}}(0)\right)$.

Case 3. $\mu_{A}(0) \geq \mu_{A^{*}}(0)$ and $v_{A}(0) \geq v_{A^{*}}(0)$.

We show $U^{t}=\left(H^{\widehat{t}}\right)^{\perp}$ and $L^{t}=\left(K^{\widehat{t}}\right)^{\perp}$, for all $t \in$ $\left(\nu_{A}(0), \mu_{A^{*}}(0)\right)$.

And if $t \in\left[v_{A^{*}}(0), v_{A}(0)\right] \cap \operatorname{Im} \mu_{A^{*}}$, then $U^{t}=V^{*}$ and $L^{t}=\{0\}$.

Case 4. $\mu_{A}(0) \leq \mu_{A^{*}}(0)$ and $v_{A}(0) \leq \nu_{A^{*}}(0)$.

We show $U^{t}=\left(H^{\widehat{t}}\right)^{\perp}$ and $L^{t}=\left(K^{\widehat{t}}\right)^{\perp}$, for all $t \in$ $\left(\nu_{A^{*}}(0), \mu_{A}(0)\right)$.

And if $t \in\left[\mu_{A}(0), \mu_{A^{*}}(0)\right] \cap \operatorname{Im} v_{A^{*}}$, then $L^{t}=V^{*}$ and $U^{t}=\{0\}$.

Hence, $A^{*}=\left(\mu_{A^{*}}, v_{A^{*}}\right)$ is an intuitionistic fuzzy subspace of $V^{*}$ since $H^{\widehat{t}}$ and $L^{\widehat{t}}$ are subspaces of $V$.

Our main theorem is as follows.

Theorem 35. Let $H$ be a finite dimensional bialgebra and let $H^{*}$ be its dual bialgebra. If $A=\left(\mu_{A}, v_{A}\right)$ is an intuitionistic fuzzy strong subbialgebra of $H$, then $A^{*}=\left(\mu_{A^{*}}, \nu_{A^{*}}\right)$ is an intuitionistic fuzzy strong subbialgebra of $H^{*}$.

Proof. $A^{*}$ is an intuitionistic fuzzy subspace of $H^{*}$ by Theorem 34. We need show that, for any $f, g \in H^{*}, \mu_{A^{*}}(f *$ $g) \geq \mu_{A^{*}}(f) \vee \mu_{A^{*}}(g), v_{A^{*}}(f * g) \leq v_{A^{*}}(f) \wedge v_{A^{*}}(g)$, and $\mu_{A^{*}}(f) \leq \mu_{A^{*}}\left(f_{i}\right) \wedge \mu_{A^{*}}\left(g_{i}\right), v_{A^{*}}(f) \geq v_{A^{*}}\left(f_{i}\right) \vee v_{A^{*}}\left(g_{i}\right)$ for all i.

Let $f, g \in H^{*}$. If $f=g=0$, the result is obvious.

If $f \neq 0$ and $g \neq 0$, we have

$$
\begin{aligned}
\mu_{A^{*}}(f * g)= & \inf \left\{v_{A}(x) \mid x \in H,(f * g)(x) \neq 0\right\} \\
\geq & \inf \left\{v_{A}\left(x_{i 1}\right) \vee v_{A}\left(x_{i 2}\right) \mid\right. \\
& \left.x_{i 1}, x_{i 2} \in H, \sum f\left(x_{i 1}\right) g\left(x_{i 2}\right) \neq 0\right\} \\
= & \inf \left\{v_{A}\left(x_{i 1}\right) \vee v_{A}\left(x_{i 2}\right) \mid\right. \\
& \left.x_{i 1}, x_{i 2} \in H, f\left(x_{i 1}\right) g\left(x_{i 2}\right) \neq 0\right\} \\
= & \inf \left\{v_{A}\left(x_{i 1}\right) \vee v_{A}\left(x_{i 2}\right) \mid\right. \\
& \left.x_{i 1}, x_{i 2} \in H, f\left(x_{i 1}\right) \neq 0, g\left(x_{i 2}\right) \neq 0\right\}
\end{aligned}
$$




$$
\begin{aligned}
= & \inf \left\{v_{A}\left(x_{i 1}\right) \mid x_{i 1} \in H, f\left(x_{i 1}\right) \neq 0\right\} \\
& \vee \inf \left\{v_{A}\left(x_{i 2}\right) \mid x_{i 2} \in H, g\left(x_{i 2}\right) \neq 0\right\} \\
\geq & \inf \left\{v_{A}(y) \mid y \in H, f(y) \neq 0\right\} \\
& \vee \inf \left\{v_{A}(z) \mid z \in H, g(z) \neq 0\right\} \\
= & \mu_{A^{*}}(f) \vee \mu_{A^{*}}(g), \\
v_{A^{*}}(f * g)= & \sup \left\{\mu_{A}(x) \mid x \in H,(f * g)(x) \neq 0\right\} \\
\leq & \sup \left\{\mu_{A}\left(x_{i 1}\right) \wedge \mu_{A}\left(x_{i 2}\right) \mid\right. \\
& \left.x_{i 1}, x_{i 2} \in H, \sum f\left(x_{i 1}\right) g\left(x_{i 2}\right) \neq 0\right\} \\
= & \sup \left\{\mu_{A}\left(x_{i 1}\right) \wedge \mu_{A}\left(x_{i 2}\right) \mid\right. \\
& \left.\quad x_{i 1}, x_{i 2} \in H, f\left(x_{i 1}\right) g\left(x_{i 2}\right) \neq 0\right\} \\
= & \sup \left\{\mu_{A}\left(x_{i 1}\right) \wedge \mu_{A}\left(x_{i 2}\right) \mid\right. \\
& x_{i 1}, x_{i 2} \in H, f\left(x_{i 1}\right) \neq 0, \\
& \left.g\left(x_{i 2}\right) \neq 0\right\} \\
= & \sup \left\{\mu_{A}\left(x_{i 1}\right) \mid x_{i 1} \in H, f\left(x_{i 1}\right) \neq 0\right\} \\
& \wedge \sup \left\{\mu_{A}\left(x_{i 2}\right) \mid x_{i 2} \in H, g\left(x_{i 2}\right) \neq 0\right\} \\
\leq & \sup \left\{\mu_{A}(y) \mid y \in H, f(y) \neq 0\right\} \\
& \wedge \sup \left\{\mu_{A}(z) \mid z \in H, g(z) \neq 0\right\} \\
= & v_{A^{*}}(f) \wedge v_{A^{*}}(g) .
\end{aligned}
$$

If $f=0$ and $g \neq 0$, then, for any $x \in H$, we have $(f *$ $g)(x)=\sum f\left(x_{1}\right) g\left(x_{2}\right)=0$. So

$$
\begin{aligned}
\mu_{A^{*}}(f * g) & =\mu_{A^{*}}(0)=\mu_{A^{*}}(f) \\
& =\sup \left\{v_{A}(x) \mid x \in H\right\} \\
& \geq \sup \left\{v_{A}(x) \mid x \in H, g(x) \neq 0\right\} \\
& \geq \inf \left\{v_{A}(x) \mid x \in H, g(x) \neq 0\right\}=\mu_{A^{*}}(g), \\
\nu_{A^{*}}(f * g) & =v_{A^{*}}(0)=v_{A^{*}}(f) \\
& =\inf \left\{\mu_{A}(x) \mid x \in H\right\} \\
& \leq \inf \left\{\mu_{A}(x) \mid x \in H, g(x) \neq 0\right\} \\
& \leq \sup \left\{\mu_{A}(x) \mid x \in H, g(x) \neq 0\right\}=v_{A^{*}}(g),
\end{aligned}
$$

which imply $\mu_{A^{*}}(f * g) \geq \mu_{A^{*}}(f) \vee \mu_{A^{*}}(g)$ and $\nu_{A^{*}}(f * g) \leq$ $v_{A^{*}}(f) \wedge v_{A^{*}}(g)$.

If $g=0$ and $f \neq 0$, the result can be proved similarly.
On the other hand, let $f \neq 0$. We have

$$
\begin{aligned}
\mu_{A^{*}}(f) & \\
= & \inf \left\{v_{A}(x) \mid x \in H, f(x) \neq 0\right\} \\
\leq & \inf \left\{v_{A}(a b) \mid x=a b \in H, f(a b) \neq 0\right\} \\
\leq & \inf \left\{v_{A}(a) \wedge v_{A}(b) \mid a, b \in H, \sum f_{i}(a) g_{i}(b) \neq 0\right\} \\
= & \inf \left\{v_{A}(a) \wedge v_{A}(b) \mid a, b \in H, f_{i}(a) g_{i}(b) \neq 0\right\} \\
= & \inf \left\{v_{A}(a) \mid a \in H, f_{i}(a) \neq 0\right\} \\
& \wedge \inf \left\{v_{A}(b) \mid b \in H, g_{i}(b) \neq 0\right\} \\
= & \mu_{A^{*}}\left(f_{i}\right) \wedge \mu_{A^{*}}\left(g_{i}\right), \\
v_{A^{*}} & (f) \\
= & \sup \left\{\mu_{A}(x) \mid x \in H, f(x) \neq 0\right\} \\
\geq & \sup \left\{\mu_{A}(a b) \mid x=a b \in H, f(a b) \neq 0\right\} \\
\geq & \sup \left\{\mu_{A}(a) \vee \mu_{A}(b) \mid a, b \in H, \sum f_{i}(a) g_{i}(b) \neq 0\right\} \\
= & \sup \left\{\mu_{A}(a) \vee \mu_{A}(b) \mid a, b \in H, f_{i}(a) g_{i}(b) \neq 0\right\} \\
= & \sup \left\{\mu_{A}(a) \mid a \in H, f_{i}(a) \neq 0\right\} \\
& \vee\left\{\mu_{A}(b) \mid b \in H, g_{i}(b) \neq 0\right\} \\
= & v_{A^{*}}\left(f_{i}\right) \vee v_{A^{*}}\left(g_{i}\right) .
\end{aligned}
$$

Let $f=0$. Noting that $f_{i}=g_{i}=0$, then $\mu_{A^{*}}(f) \leq$ $\mu_{A^{*}}\left(f_{i}\right) \wedge \mu_{A^{*}}\left(g_{i}\right)$ and $v_{A^{*}}(f) \geq v_{A^{*}}\left(f_{i}\right) \vee v_{A^{*}}\left(g_{i}\right)$.

Hence, $A^{*}=\left(\mu_{A^{*}}, v_{A^{*}}\right)$ is an intuitionistic fuzzy strong subbialgebra of $H^{*}$.

Remark 36. (1) If $A=\left(\mu_{A}, v_{A}\right)$ is an intuitionistic fuzzy subcoalgebra of $H$, then $A^{*}=\left(\mu_{A^{*}}, \nu_{A^{*}}\right)$ is an intuitionistic fuzzy ideal of $H^{*}$, and it is an intuitionistic fuzzy subalgebra of $H^{*}$.

(2) If $A=\left(\mu_{A}, v_{A}\right)$ is an intuitionistic fuzzy ideal of $H$, then $A^{*}=\left(\mu_{A^{*}}, v_{A^{*}}\right)$ is an intuitionistic fuzzy subcoalgebra of $H^{*}$.

Question 1. If $A=\left(\mu_{A}, v_{A}\right)$ is an intuitionistic fuzzy subalgebra of $H$, is the result true?

Example 37. See Example 17 and we assume $k$ is finite dimension.

We calculate

$$
\begin{aligned}
& \mu_{A^{*}}(f)= \begin{cases}0.2 \wedge 0.4 & \text { if } f \neq 0 \\
0.4 & \text { if } f=0,\end{cases} \\
& \nu_{A^{*}}(f)= \begin{cases}0.3 \vee 0.5 & \text { if } f \neq 0 \\
0.3 & \text { if } f=0 .\end{cases}
\end{aligned}
$$


Since $A=\left(\mu_{A}, v_{A}\right)$ is an intuitionistic fuzzy subbialgebra, we get $\mu_{A^{*}}(f) \leq \mu_{A^{*}}\left(f_{i}\right) \vee \mu_{A^{*}}\left(g_{i}\right)$ and $\nu_{A^{*}}(f) \geq v_{A^{*}}\left(f_{i}\right) \wedge$ $v_{A^{*}}\left(g_{i}\right)$ following the proof of Theorem 35. If $\mu_{A^{*}}(f)=0.4$, $\mu_{A^{*}}\left(f_{i}\right)=0.2$ and $\mu_{A^{*}}\left(g_{i}\right)=0.4$, then $\mu_{A^{*}}(f) \leq \mu_{A^{*}}\left(f_{i}\right) \vee$ $\mu_{A^{*}}\left(g_{i}\right)$. However, $\mu_{A^{*}}(f)>\mu_{A^{*}}\left(f_{i}\right) \wedge \mu_{A^{*}}\left(g_{i}\right)$; this shows that it is not an intuitionistic fuzzy subcoalgebra of $k^{*}$. Hence, Theorem 35 is not true when $A=\left(\mu_{A}, v_{A}\right)$ is an intuitionistic fuzzy subbialgebra.

\section{Conclusion}

An intuitionistic fuzzy subbialgebra of a bialgebra is defined with an intuitionistic fuzzy subalgebra structure and with an intuitionistic fuzzy subcoalgebra structure. Since the main problem in uncertainty mathematics is how to carry out the ordinary concepts to the uncertainty case and the difficulty lies in how to pick out the rational generalization from the large number of available approaches, the key of studying the dual of intuitionistic fuzzy subbialgebras is to give a rational definition. We define the dual of an intuitionistic fuzzy subspace which generalizes the dual of fuzzy subspaces nontrivially.

Hopf algebra is a bialgebra with antipode which is ubiquity in virtually all fields of mathematics. We will focus on considering Hopf algebras in the intuitionistic fuzzy settings as a continuation of this paper in the future. Also, we will consider bialgebras in rough sets and soft sets. Our obtained results probably can be applied in various fields such as artificial intelligence, signal processing, multiagent systems, pattern recognition, robotics, expert systems, medical diagnosis, and engineering.

\section{Conflict of Interests}

No conflict of interests exits in the submission of this paper.

\section{Acknowledgments}

This project is supported by the National Natural Science Foundation of China (Grant no. 11126301) and the Promotive Research Fund for Young and Middle-aged Scientists of Shandong Province (no. BS2011SF002).

\section{References}

[1] S. Dăscălescu, C. Năstăsescu, and . Raianu, Hopf Algebras, vol. 235 of Monographs and Textbooks in Pure and Applied Mathematics, Marcel Dekker, New York, NY, USA, 2001.

[2] L. A. Zadeh, "Fuzzy sets," Information and Computation, vol. 8, pp. 338-353, 1965.

[3] K. T. Atanassov, "Intuitionistic fuzzy sets," Fuzzy Sets and Systems, vol. 20, no. 1, pp. 87-96, 1986.

[4] K. T. Atanassov, "New operations defined over the intuitionistic fuzzy sets," Fuzzy Sets and Systems, vol. 61, no. 2, pp. 137-142, 1994.

[5] R. Biswas, "Intuitionistic fuzzy subgroups," Mathematics Forum, vol. 10, pp. 37-46, 1989.
[6] B. Banerjee and D. Kr. Basnet, "Intuitionistic fuzzy subrings and ideals," Journal of Fuzzy Mathematics, vol. 11, no. 1, pp. 139-155, 2003.

[7] K. Hur, H. W. Kang, and H. K. Song, "Intuitionistic fuzzy subgroups and subrings," Honam Mathematical Journal, vol. 25, no. 1, pp. 19-41, 2003.

[8] K. Hur, S. Y. Jang, and H. W. Kang, "Intuitionistic fuzzy ideals of a ring," Journal of the Korea Society of Mathematical Education, vol. 12, no. 3, pp. 193-209, 2005.

[9] Y. B. Jun, M. A. Öztürk, and C. H. Park, "Intuitionistic nil radicals of intuitionistic fuzzy ideals and Euclidean intuitionistic fuzzy ideals in rings," Information Sciences, vol. 177, no. 21, pp. 4662-4677, 2007.

[10] M. Akram and W. A. Dudek, "Intuitionistic fuzzy left k-ideals of semirings," Soft Computing, vol. 12, pp. 881-890, 2008.

[11] B. Davvaz, W. A. Dudek, and Y. B. Jun, "Intuitionistic fuzzy $H_{v^{-}}$ submodules," Information Sciences, vol. 176, no. 3, pp. 285-300, 2006.

[12] W. A. Dudek, B. Davvaz, and Y. B. Jun, "On intuitionistic fuzzy sub-hyperquasigroups of hyperquasigroups," Information Sciences, vol. 170, no. 2-4, pp. 251-262, 2005.

[13] W. A. Dudek, J. Zhan, and B. Davvaz, "On intuitionistic (S, T)-fuzzy hypergroups," Soft Computing, vol. 12, pp. 1229-1238, 2008.

[14] B. Davvaz and V. Leoreanu-Fotea, "Intuitionistic fuzzy $n$ ary hypergroups," Journal of Multiple-Valued Logic and Soft Computing, vol. 16, no. 1-2, pp. 87-104, 2010.

[15] B. Davvaz, P. Corsini, and V. Leoreanu-Fotea, "Atanassov's intuitionistic $(S, T)$-fuzzy $n$-ary sub-hypergroups and their properties," Information Sciences, vol. 179, no. 5, pp. 654-666, 2009.

[16] I. Cristea and B. Davvaz, "Atanassov's intuitionistic fuzzy grade of hypergroups," Information Sciences, vol. 180, no. 8, pp. 15061517, 2010.

[17] W. Chen and S. Zhang, "Intuitionistic fuzzy Lie subsuperalgebras and intuitionistic fuzzy ideals," Computers \& Mathematics with Applications, vol. 58, no. 8, pp. 1645-1661, 2009.

[18] W. Chen, "Intuitionistic fuzzy quotient Lie superalgebras," International Journal of Fuzzy Systems, vol. 12, no. 4, pp. 330339, 2010.

[19] W. Chen, "Fuzzy subcoalgebras and duality" Bulletin of the Malaysian Mathematical Sciences Society, vol. 32, no. 3, pp. 283294, 2009.

[20] W. Chen and W. Ge, "Fuzzy subbialgebras and duality," Utilitas Mathematica, vol. 87, pp. 13-20, 2012.

[21] W. Chen, M. Akram, and Y. Guan, "Intuitionistic fuzzy subcoalgebras of coalgebras," Ars Combinatoria, vol. 106, pp. 423-434, 2012.

[22] K. S. Abdukhalikov, M. S. Tulenbaev, and U. U. Umirbaev, “On fuzzy subalgebras," Fuzzy Sets and Systems, vol. 93, no. 2, pp. 257-262, 1998.

[23] W. Chen and Y. Guan, "The dual of generalized fuzzy subspaces," Journal of Applied Mathematics, vol. 2012, Article ID 932014, 9 pages, 2012. 


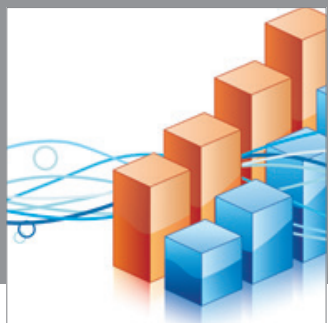

Advances in

Operations Research

mansans

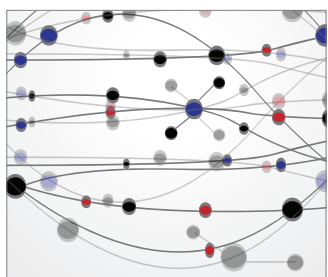

The Scientific World Journal
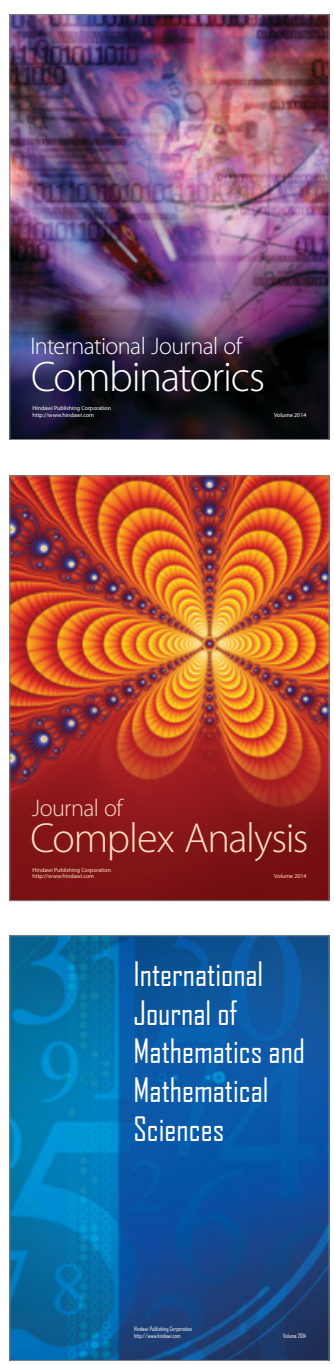
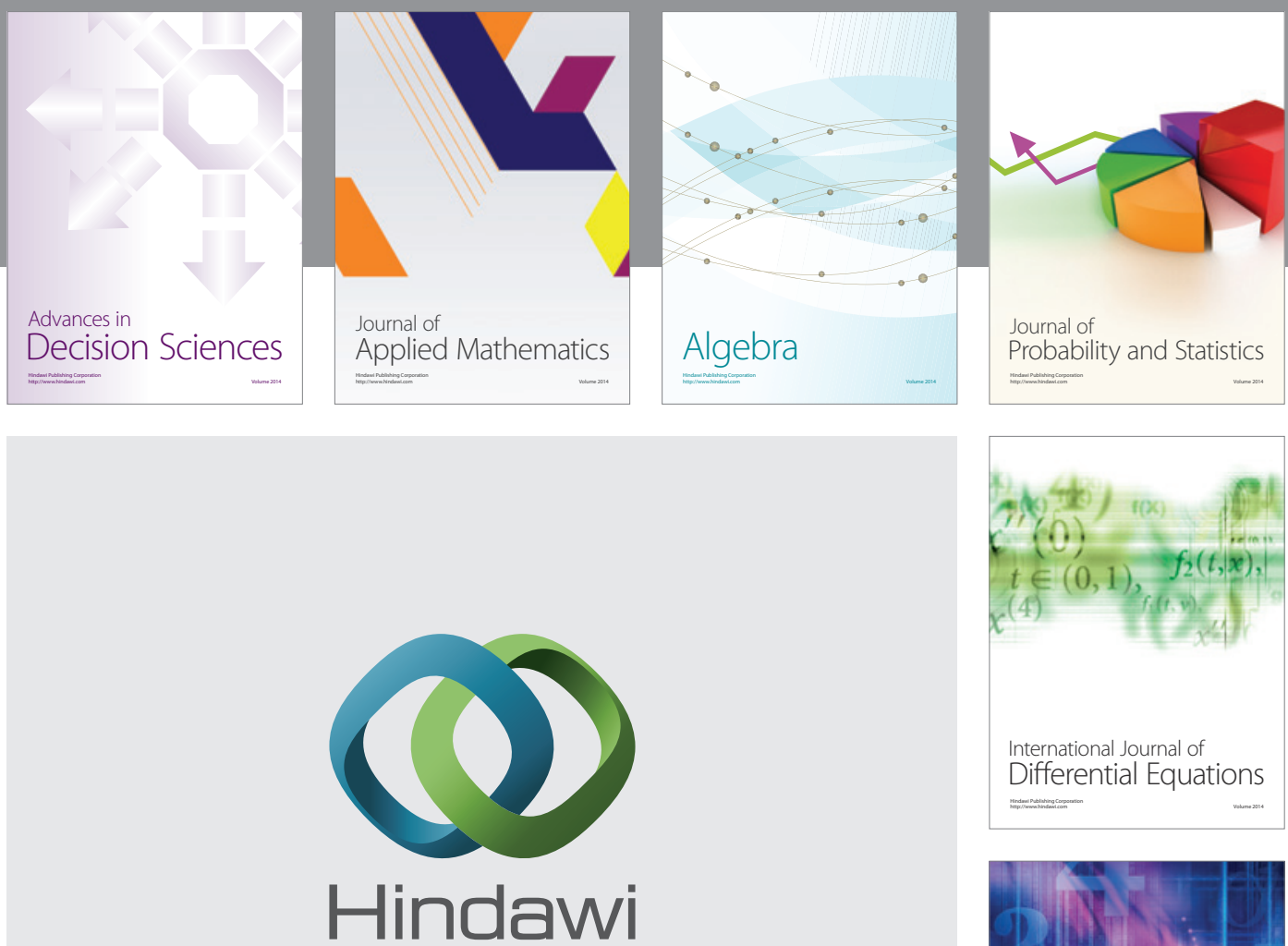

Submit your manuscripts at http://www.hindawi.com
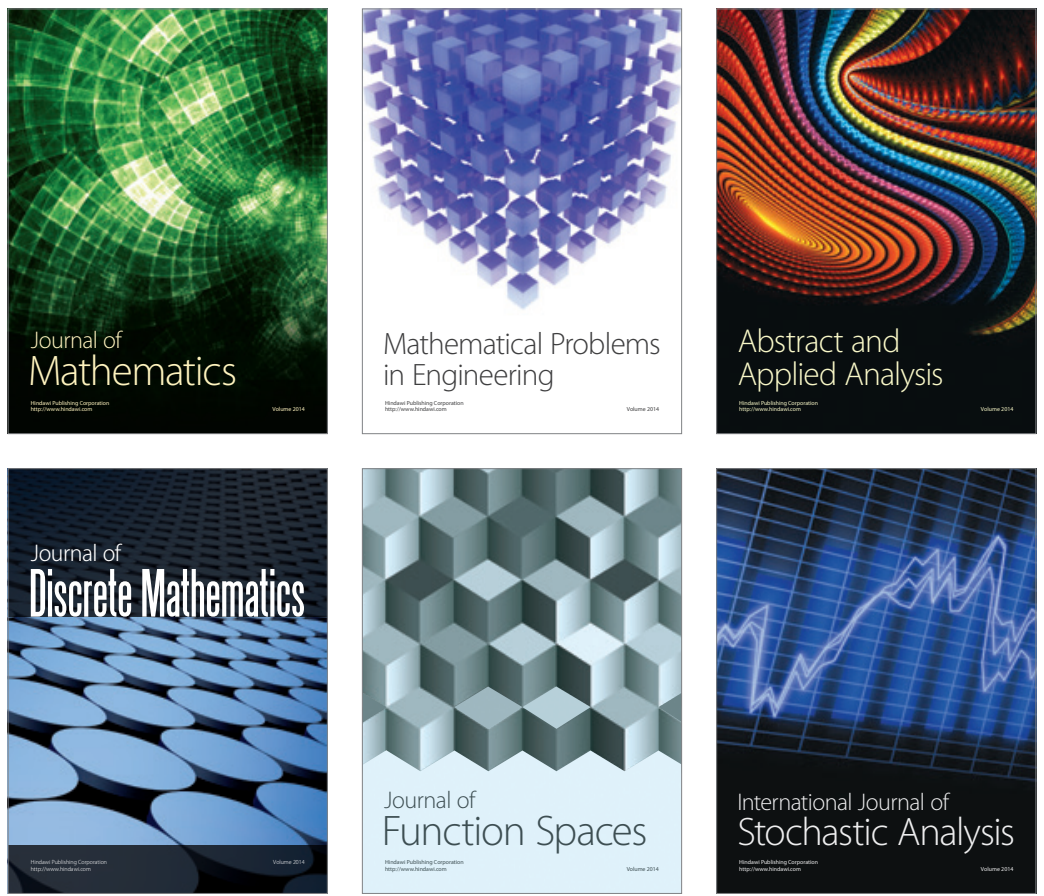

Journal of

Function Spaces

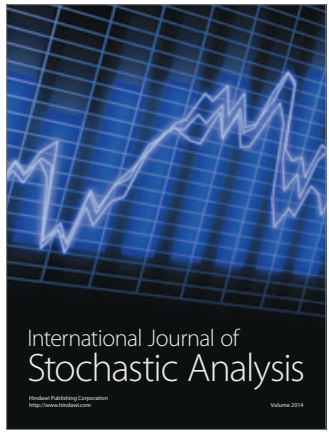

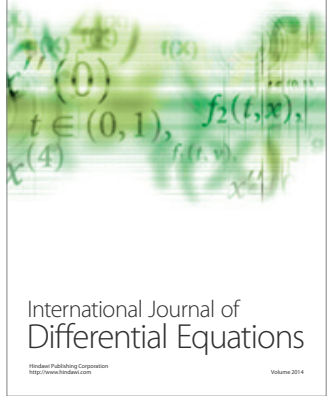
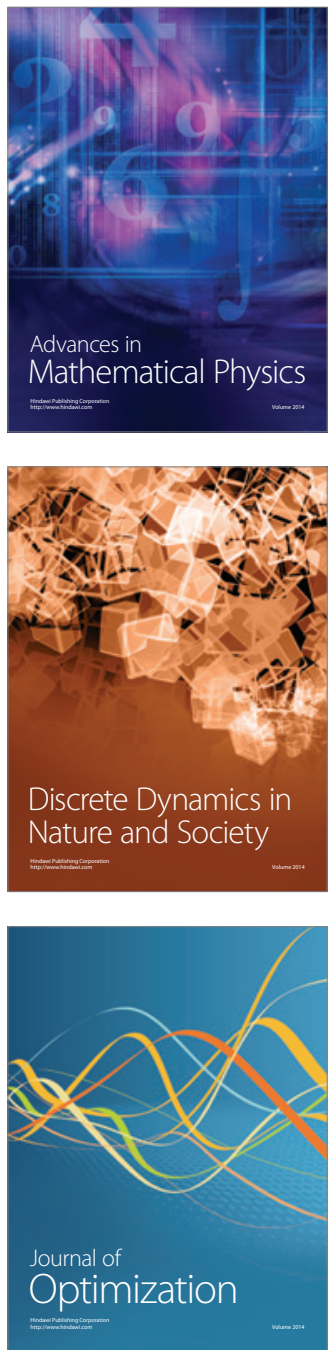